\title{
Implementation and Initial Analysis of a Laboratory-Based Weekly Biosurveillance System, Provence-Alpes-Côte d'Azur, France
}

\author{
Michael Huart, Gabriel Bedubourg, Cédric Abat, Philippe Colson, Jean Marc Rolain, \\ Hervé Chaudet, Pierre Edouard Fournier, Didier Raoult, Xavier Deparis
}

We describe the implementation of an automated infectious disease surveillance system that uses data collected from 210 microbiologic laboratories throughout the ProvenceAlpes-Côte d'Azur region in France. Each week, these facilities report bacterial species that have been isolated from patients in their area. An alarm is triggered whenever the case count for a bacterial species infection exceeds 2 SDs of the historical mean for that species at the participating laboratory. At its inception in July 2013, the system monitored 611 bacterial species. During July 1, 2013-March 20, 2016 , weekly analyses of incoming surveillance data generated 34 alarms signaling possible infectious disease outbreaks; after investigation, 14 (41\%) of these alarms resulted in health alerts declared by the regional health authority. We are currently improving the system by developing an Internet-based surveillance platform and extending our surveillance to include more laboratories in the region.

$\mathrm{D}$ uring the second half of the 20th century, infectious diseases were considered a public health concern belonging to the past (1). However, despite some decrease in epidemiologic importance (2), infectious diseases remain a major cause of illness and lead to $>25 \%$ of annual deaths (3-5). To ensure the timely detection of infectious diseases,

Author affiliations: Centre d'Epidémiologie et de Santé Publique des Armées, Marseille, France (M. Huart, G. Bedubourg,

X. Deparis); Unité de Recherche sur les Maladies Infectieuses et Tropicales Emergentes, Aix-Marseille Université, Marseille (M. Huart, C. Abat, P. Colson, J.M. Rolain, H. Chaudet,

P.E. Fournier, D. Raoult); Fondation Institut Hospitalo-Universitaire Méditerranée Infection-Assistance Publique-Hôpitaux de Marseille, Marseille (M. Huart, C. Abat, P. Colson, J.M. Rolain, P.E. Fournier, D. Raoult); Sciences Economiques et Sociales de la Santé et Traitement de l'Information Médicale, Aix Marseille Université, Marseille (M. Huart, C. Abat, P. Colson, J.M. Rolain, P.E. Fournier, D. Raoult)

DOI: http://dx.doi.org/10.3201/eid2304.161399 health authorities have proposed the implementation of health surveillance systems. Historically, surveillance started with the use of mortality and morbidity data for public health purposes, which was first proposed by John Graunt in 1657 (6). The concept of surveillance has evolved over the centuries, and surveillance is now conducted mainly through the monitoring of symptoms and syndromes. During the 20th century, an expansion of the surveillance concept occurred with the emergence of numerous surveillance systems $(4,7)$. Epidemiologic surveillance came to be known for 3 basic characteristics: systematic collection of data, consolidation and analysis of the collected data, and dissemination of information through narrative epidemiologic reports (3). Since 2001, because of the threat of bioterrorist attacks and the emergence and reemergence of infectious diseases, such as the recent Ebola outbreak in West Africa, interest in the methods for detection of infectious diseases has increased $(4,8)$.

In the Assistance Publique-Hôpitaux de Marseille (AP-HM) public hospital network in Marseille, France, weekly automated epidemiologic surveillance systems have been implemented since $2002(9,10)$. The objectives of these systems are to analyze clinical data produced by the microbiologic laboratories of 4 public hospitals in Marseille. The first program implemented, the Epidemiologic Surveillance and Alert Based on Microbiological Data, has monitored more than 293 infectious disease-related items on a weekly basis since November 2002 (9), including 38 clinical samples, 86 pathogens, 79 diagnosis tests, and 39 antimicrobial-resistance patterns. After the introduction of this system, several other systems based on a previously described historical database (11) were set up, such as the Bacterial Real-Time Laboratory-Based Surveillance System (BALYSES) and the Marseille Antibiotic Resistance Surveillance System (MARSS) (10). The latter 2 systems have routinely operated in the AP-HM network since 2013. During May 21, 2013-June 4, 2014, BALYSES detected 
21 alarms (triggered when the number of cases of an infectious disease exceeds the statistic threshold), and MARSS detected 31. For BALYSES, 5 alarms either were escalated into alerts after further investigation or led to official reports to the Regional Health Agency (Agence Régionale de Santé [ARS]) of PACA, and for MARSS, 16 alarms led to official reports (10).

In July 2013, we aimed to expand the epidemiologic surveillance implemented in the AP-HM network to the entire PACA region by developing a new specific surveillance tool. This tool was developed in collaboration with the Armed Forces Center for Epidemiology and Public Health in France (CESPA). Until that time, no laboratory network in France had been implemented to monitor so many infectious disease-related events (12). Several other epidemiologic surveillance networks of microbiologic laboratories exist worldwide, such as the system set up by $\mathrm{C}$. Paddy Farrington et al. in England and Wales (13) or the "Vigie" network in Belgium (14).

The PACA region is a population hub with many airports and ports with large flows of migrants and travelers. These population flows could bring infectious disease into the PACA region. Therefore, implementation of such a biosurveillance system based on previously unused data from microbiologic laboratories in the PACA region was expected to improve infectious diseases surveillance. Here we describe the procedure for implementing this biosurveillance system in and the initial results obtained from July 2013 through the end of March 2016.

\section{Implementing a Biosurveillance System}

\section{Study Setting}

The PACA region is located in southeastern France and is the third most populated region in the country, with $\approx 4.9$ million inhabitants $(\approx 7.5 \%$ of the total French population) (15). Several major cities are located in the region, such as Marseille, Toulon, and Nice, with 850,636, 163,974, and 343,064 inhabitants, respectively, in 2014 (15). The PACA region, which borders on Italy, is an important hub, with large population flows from North Africa across the Mediterranean Sea. In 2015, the PACA region had $\approx 611$ private or public microbiologic laboratories according to ARS (ARS, unpub. data). Some of these laboratories have formed groups or networks, which can consist of up to 70 laboratories (Table 1). The geographic coverage of the laboratories included in our biosurveillance network is homogeneous over the region (Figure 1).

\section{Biosurveillance System}

We created a biosurveillance system, the PACA Surveillance Epidemiologic System (PACASurvE), capable of collecting, standardizing, and computing the laboratory results produced by public (i.e., hospital-affiliated) and private-sector microbiology laboratories located in the PACA region every week. The system's objectives are to provide early detection capability and an initial description of possible infectious disease threats $(10,16)$; accordingly, the system is designed to issue alarms if an outbreak is detected or if a single case of a rare but severe infectious disease or an unknown infectious agent is discovered.

PACASurvE is Internet-based and uses Excel software (Microsoft, Redmond, WA, USA) for data collection and management and $\mathrm{R}$ version 3.0.1 software (17) for analysis. The system was implemented and has been routinely used since July 2013. Public hospital and private sector laboratories of the PACA region were invited to participate in the surveillance network. Fifteen institutions

Table 1. Selected characteristics of facilities participating in the Provence Alpes Côte d'Azur Surveillance Epidemiologic System, France, July 1, 2013-March 20, 2016*

\begin{tabular}{|c|c|c|c|c|c|c|}
\hline Facility & Status & Geographic area & $\begin{array}{l}\text { No. laboratories, } \\
N=210\end{array}$ & $\begin{array}{l}\text { Wks since } \\
\text { launch }\end{array}$ & $\begin{array}{l}\text { Continuous or } \\
\text { discontinuous }\end{array}$ & $\begin{array}{l}\text { Start date of } \\
\text { surveillance }\end{array}$ \\
\hline LABM Labazur Provence & Private & $\begin{array}{l}\text { Bouches-du-Rhône, } \\
\text { Var, Vaucluse }\end{array}$ & 26 & 142 & Continuous & 2013 Jul 1 \\
\hline LABM Alphabio & Private & Marseille & 17 & 142 & Continuous & 2013 Jul 1 \\
\hline Clinique La Casamance & Public & Marseille & 1 & 139 & Continuous & 2013 Jul 22 \\
\hline LABM Analys & Private & Boûches-du-Rhône & 19 & 127 & Discontinuous & 2013 Oct 14 \\
\hline $\mathrm{CH}$ Aix-en-Provence & Public & Aix-en-Provence & 1 & 122 & Discontinuous & 2013 Nov 18 \\
\hline CHU Nice & Public & Nice & 1 & 118 & Continuous & 2013 Dec 16 \\
\hline $\mathrm{CH}$ Martigues & Public & Martigues & 1 & 114 & Continuous & 2014 Jan 13 \\
\hline $\mathrm{CH}$ Salon-de-Provence & Public & Salon-de-Provence & 1 & 109 & Discontinuous & 2014 Feb 14 \\
\hline Hôpital Inter-Armées, Laveran & Public & Marseille & 1 & 100 & Discontinuous & 2014 Apr 21 \\
\hline LABM Cerba & Private & PACA & 70 & 63 & Continuous & 2015 Jan 5 \\
\hline Hôpital Saint-Joseph & Public & Marseille & 1 & 59 & Discontinuous & 2015 Feb 3 \\
\hline LABM BioAlliance & Private & Marseille & 21 & 42 & Continuous & 2015 Jun 1 \\
\hline LABM Labazur Nice & Private & Alpes Maritimes & 28 & 23 & Continuous & 2015 Oct 12 \\
\hline $\mathrm{CH}$ Dignes & Public & Dignes & 1 & 11 & Discontinuous & 2016 Jan 1 \\
\hline LABM Barla & Private & Nice & 21 & 7 & Continuous & 2016 Feb 3 \\
\hline
\end{tabular}

${ }^{*} \mathrm{CH}$, Centre Hospitalier (Central Hospital); CHU, Centre Hospitalier Universitaire (Central University Hospital); LABM, Laboratoire de Biologie Médicale (Medical Laboratory); PACA, Provence Alpes Côte d'Azur. 


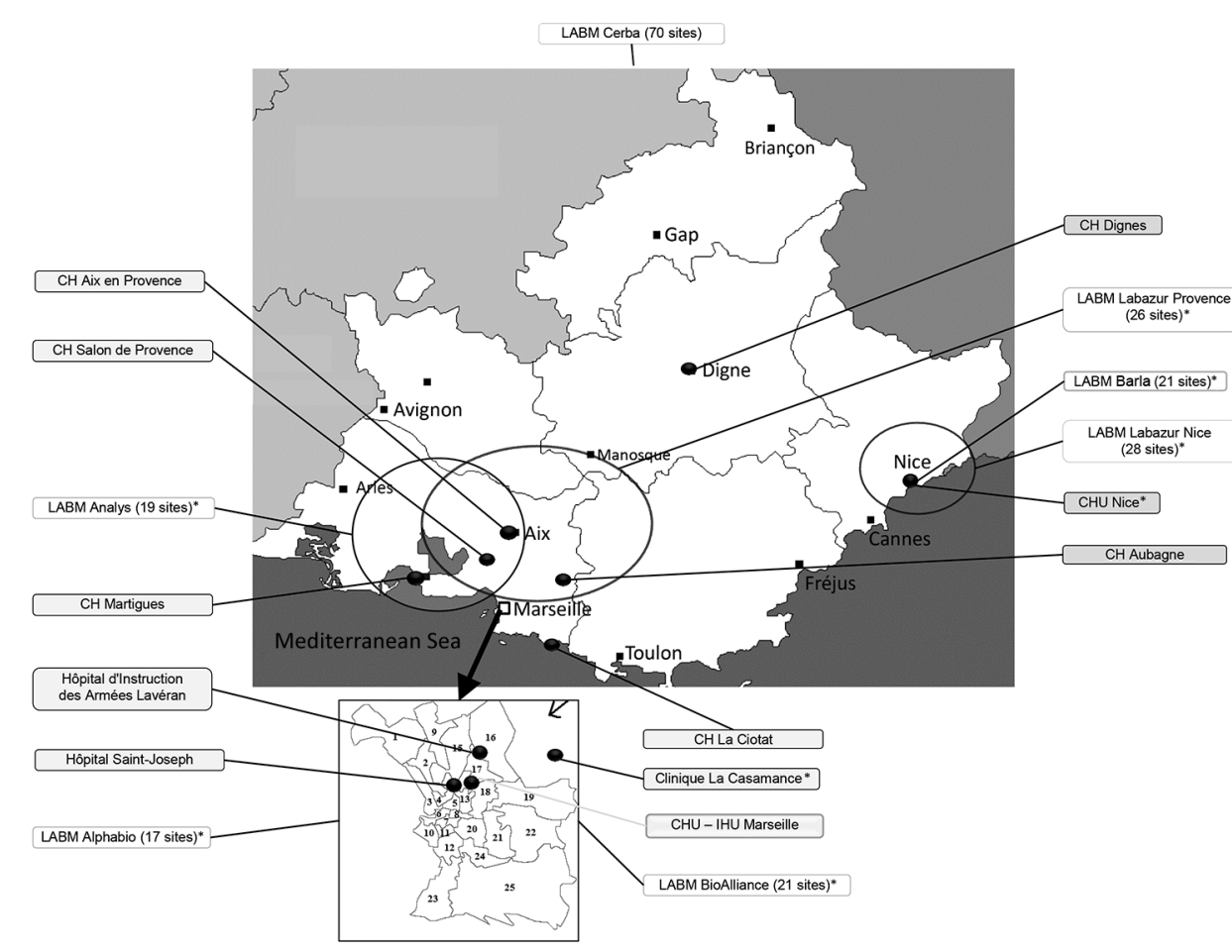

Figure 1. Laboratories participating in the Provence Alpes Côte d'Azur Surveillance Epidemiologic System, France, July 1, 2013-March 20, 2016. Black dots indicate participating laboratories; black boxes indicate public laboratories; text labels indicate private laboratories and areas of activity. Asterisks $\left({ }^{*}\right)$ denote laboratories using matrix-assisted laser desorption/ionization timeof-flight mass spectrometry for identification of species; all other laboratories shown use biochemical bacterial identification. $\mathrm{CH}$, Centre Hospitalier (Central Hospital); $\mathrm{CHU}$, Centre Hospitalier Universitaire (Central University Hospital); IHU, Institut HospitaloUniversitaire (Hospital-University Institute); LABM, Laboratoire de Biologie Médicale (Medical Laboratory).

were first selected among the leading laboratories in terms of volume of activity to rapidly achieve a better geographic representation of PACA (Figure 1). Their participation was based on several criteria proposed in the literature and relevant to the implementation of our network, including those described by Walckiers et al. (14): participation of laboratories on an unpaid and voluntary basis, participation of microbiologic laboratories, anonymity of data, and a standard, predefined frequency for data collection (14).

After identifying the participating institutions, the second task was to define which events should be monitored and their respective definitions, which were transmitted to all laboratories. Data collected included information on bacterial identification and virologic, bacteriologic, mycologic, and parasitologic laboratory results. Currently, PACASurvE is particularly geared toward the monitoring of bacterial species.

We defined a case as illness in a patient from whom $\geq 1$ bacterial species was isolated and confirmed. Two different bacterial species isolated from samples collected from the same patient resulted in 2 reported cases. The samples without bacterial identification were considered to be negative. Laboratories were free to use the microbiologic methods of their choice to identify bacterial species, including PCR, matrix-assisted laser desorption/ ionization time-of-flight mass spectrometry, or conventional phenotypic methods (e.g., Gram coloration and API galleries).
A weekly coordination meeting, which included members from AP-HM and CESPA, was defined to optimize data collection and organize analyses and feedback. Currently, PACASurvE is included in a comprehensive biosurveillance system at the AP-HM network with the other epidemiologic surveillance systems previously described (Figure 2).

\section{Data Flow, Analyses, and Feedback}

All the steps of data flow, analyses, and feedback were mapped (Figure 2). Every week, biologists at participating institutions sent a report of new cases, in the form of anonymized data contained in Excel spreadsheets or encrypted PDFs, to the system coordinator.

The first step before analysis was validating and standardizing the reported data, which were checked, cleaned, and deduplicated. The search for duplicates was performed weekly according to the unique patient identifier and the isolated microorganism, and data were then automatically compared with a thesaurus of all identified bacterial species (10). All analysis steps (e.g., deduplication, merging of data from different laboratories, statistical analysis, and visualization tools production) were performed automatically by using a specific algorithm written in a Visual Basic (Microsoft) script for Excel.

To detect outbreaks as early as possible, an alarm was triggered when the weekly count of cases for a bacterial species was higher than 2 SDs of the mean of historical data since the beginning of surveillance for each laboratory (10). 
After 6 months, once the collection procedures were stabilized, the C1-mild epidemics detection method used by the Early Aberration Reporting System $(18,19)$ was performed. This method enables the detection of outbreaks on a dataset with limited historical data ( $>7$ weeks). Both of these statistical methods operate in parallel at the Institut HospitaloUniversitaire Méditérannée Infection and CESPA.

A statistical alarm is triggered if the observed value is significantly different from the expected value (16). After checking biologic criteria, alarms were assessed as confirmed or unconfirmed by senior biologists during the weekly AP-HM epidemiologic surveillance meeting. Alarms that were escalated into an alert (after further investigations that included diagnosis confirmation and descriptive analysis of cases in terms of time, place, and population) led to further epidemiologic investigation, which then had to be declared to ARS if a real outbreak was confirmed. Specific countermeasures also had to be implemented, such as patient isolation, implementation of specific care protocols, or a large scale information campaign (Figure 2). For feedback, a weekly epidemiologic report was addressed to all participating laboratories, AP-HM department officials, CESPA, ARS, and the Interregional Epidemiology Unit (otherwise known as CIRE).

\section{Results}

\section{Scalability of the System}

In July 2013, when PACASurvE started, 3 main structures that collected data from 44 laboratories sent their anonymized data to the network coordinator every Monday. In March 2016, a total of 15 participating institutions were included in the biosurveillance system (Table 1). Several participated irregularly; 2 (Centre Hospitalier Dignes and Centre Hospitalier Aix en Provence) transmitted a common declaration file. Currently, PACASurvE includes 8 public and 7 private sector participating institutions (Figure 1), representing a total of 210 laboratories (34.4\% of all laboratories in the PACA region).

\section{Description of Collected Cases}

An average of 14,000 cases (positive and negative) were reported every week. Since the beginning of the biosurveillance system, 217,621 bacterial infections have been reported by participating structures (i.e., $\approx 1,532$ confirmed cases per week). These identifications resulted from the analysis of an estimated 315,000 urine samples, 140,000 blood cultures, 6,700 respiratory specimens, 32,000 stool samples, 4,400 cerebrospinal fluid samples, and 176,000 serologic examinations.
Figure 2. Flow diagram of all epidemiologic surveillance systems implemented by the Institut Hospitalo-Universitaire Méditérannée Infection, Assistance Publique-Hôpitaux de Marseille, France. ARS, Agence Régionale de Santé (Regional Health Agency); BALYSES, Bacterial Real-Time Laboratory-Based Surveillance System; CDS, Centre de Santé (Health Center); CHG, Centre Hospitalier Général (General Hospital Center); CHU, Centre Hospitalier Universitaire (Central University Hospital); CLIN, Comité de Lutte contre les Infections Nosocomiales (Committee for the Fight Against Nosocomial Infections); DGS, Direction Générale de la Santé (Directorate General for Health); EPIMIC, Epidemiologic Surveillance and Alert Based on Microbiological Data; IHU/ AP-HM, Institut HospitaloUniversitaire/Assistance Publique-Hôpitaux de Marseille; INVS, Institut Nationale de Veille Sanitaire (National Institute for Public Health Surveillance); LABM, Laboratoire de Biologie Médicale (Medical Laboratory); MARSS, Marseille Antibiotic Resistance Surveillance System; PACASurvE, Provence Alpes Côte d'Azur Surveillance Epidemiologic System. Diagram is based on the workflow described by Abat et Al. 2013 (10). 
Table 2. Ten bacterial species with the most alarms triggered by the Provence Alpes Côte d'Azur Surveillance Epidemiologic System, France, July 1, 2013-March 20, 2016*

\begin{tabular}{lcc}
\hline Bacterial species & $\begin{array}{c}\text { No. alarms } \\
\text { total }\end{array}$ & $\begin{array}{c}\text { Average weekly } \\
\text { no. alarms }\end{array}$ \\
\hline Pseudomonas putida & 87 & 0.6 \\
Stenotrophomonas maltophilia & 82 & 0.6 \\
Neisseria gonorrhoeae & 78 & 0.6 \\
Hafnia alvei & 74 & 0.5 \\
Enterobacter aerogenes & 72 & 0.5 \\
Staphylococcus capitis & 72 & 0.5 \\
Staphylococcus lugdunensis & 70 & 0.5 \\
Streptococcus constellatus & 68 & 0.5 \\
Staphylococcus haemolyticus & 66 & 0.5 \\
Haemophilus parainfluenzae & 65 & 0.5 \\
*Total no. alarms for the entire system during this period was 5,915 \\
(averaging 42 alarms weekly).
\end{tabular}

\section{Thesaurus}

Data were compared automatically to a thesaurus that included 611 bacterial species at the time of the system's inception in 2013 (10). Currently, the number of bacterial species is 673 .

Top 10 Identified Bacteria in PACASurvE and Biodiversity We ranked the overall top 10 bacterial species isolated since the beginning of the biosurveillance system and the top 10 per laboratory. The 10 most frequently reported bacterial species in PACASurvE represented 181,241 identifications (83.1\% of total cases) (online Technical Appendix, https://wwwnc.cdc.gov/EID/article/23/4/16-1399Techapp1.xlsx). Escherichia coli infections were the most frequently reported cases in all the laboratories. For others species, diversity increased when frequency decreased (online Technical Appendix).

\section{Bacterial Species Specifically Isolated by PACASurvE} PACASurvE has also made it possible to identify bacterial species that were unknown in the initial thesaurus of APHM (10). A total of 12 bacterial species have been isolated and added to this thesaurus: Citrobacter werkmanii, Kluyvera cryocrescens, Lactobacillus brevis, Streptococcus pluranimalium, Paenibacillus peoriae, Rhodotorula minuta, Cronobacter malonaticus, Paenibacillus durus, Rhodotorula mucilaginosa, Rhizobium radiobacter, Buttiauxella agrestis, and Plesiomonas shigelloides.

\section{Alarms and Alerts}

The biosurveillance system issued alarms every week after analysis. These alarms were triggered by an increasing number of reported cases for some bacterial species. The system has issued 5,915 alarms since July 2013, averaging 2,160 alarms per year and 41 alarms per week. Since July 2013, after analysis at the weekly coordination meetings, 34 alarms required further investigations after validation by a senior epidemiologist or a biologist, and 14 (41\%) of those were escalated to an alert. We ranked the 10 bacterial species that have triggered the largest number of alarms since the beginning of the surveillance network (Table 2).

Fourteen notifications have been reported to ARS as outbreaks, which were caused by the following bacterial species: Enterococcus faecalis, Clostridium difficile (serotype $\mathrm{O} 27$ and non-O27), Escherichia coli, Acinetobacter radioresistens, Serratia marcescens, Enterobacter aerogenes, Proteus penneri, Streptococcus pyogenes, and Streptococcus aerogenes. All these epidemiologic events were identified by PACASurvE and confirmed after further detection by other surveillance systems. Other alarms were declared for laboratories or hospitals involved in investigations. These investigations did not necessarily lead to an alert but more often to an internal investigation.

Alarms regarding an E. faecalis outbreak were issued by PACASurvE in March 2015 (20), largely because of an increase in the number of declared cases of $E$. faecalis isolated in urine samples in Marseille and surroundings areas. That outbreak was reported to ARS, and investigations are still ongoing to find out if a single or multiple clones were responsible.

Alarms regarding $C$. difficile were issued in the hospital monitoring system BALYSES and among private-sector laboratories in the PACASurvE system. These alarms led to a further investigation into $C$. difficile-related illness and death in patients. We found the presence of a hypervirulent strain (O27) (21). However, this strain does not account for all the deaths attributable to $C$. difficile (22) because other strains of this bacterial species exist. Therefore, an alert was issued to ARS, which led to specific countermeasures (e.g., systematic screening, isolation of patients, transport to the infectious diseases unit at Hôpital Nord in Marseille, and establishment of a specific treatment protocol with early fecal transplant) (23).

\section{Feedback and Network Management}

The feedback bulletin was set up to keep all participants in the surveillance network informed. It consisted of a presentation with 2-3 slides per participating laboratory, with a summary of their declared activity during the previous week. It was accompanied by an email newsletter with information on the main alarms that led to further investigations and interpretation by a college of experts. The weekly epidemiologic bulletin was also available on the website of the Institut Hospitalo-Universitaire Méditérannée Infection (http://www.mediterranee-infection.com/article. php?larub=23\&titre=surveillance-epidemiologique).

\section{Discussion}

Since July 2013, we have been operating a biosurveillance system based on a network of clinical microbiology laboratories in the PACA region to monitor infectious diseases, especially those attributable to bacterial species. 
To date, to our knowledge, the PACASurvE network is unique in France. It is a collaborative system (one relying on the participation of private and hospital laboratories) which is different from BALYSES (a hospital system) (10). Similar biosurveillance networks were developed in Belgium $(14,24)$, the United Kingdom (13), and the United States, where the Laboratory Response Network was implemented by the Centers for Disease Control and Prevention in 1999 (25). In Belgium, a sentinel network using microbiology laboratory data was created for the weekly monitoring of selected pathogens $(24,26)$. In contrast, PACASurvE focuses on the monitoring of 673 bacterial species.

In the United States, the Laboratory Response Network was implemented to build a network of laboratories that can respond to biologic and chemical emergencies. Our surveillance system only focused on biologic threats and was intended for the early detection of infectious disease outbreaks on a weekly basis.

After initial difficulties in enrolling laboratories, the biosurveillance system now functions regularly. The system has generated 14 alarms that have been investigated and reported to ARS. These alarms made it possible to detect actual outbreaks and helped to develop effective countermeasures, as in the case of the $C$. difficile (23) and $E$. faecalis (20) outbreaks.

This system has several strengths and some limitations. The first and main strength of the system is that it can easily be replicated, thanks to its low implementation cost and its use of Excel software. The use of this software allowed the system to be set up rapidly, and any necessary modifications can be made easily, compared with the software used by other surveillance systems, such as Real Time Outbreak and Disease Surveillance (27). This simplicity could allow it to be implemented in developing countries.

Continuous improvement also is a major strength of PACASurvE. The number of laboratories increases regularly, which improves the representativeness of the PACA region. Improving geographic representativeness is important for the purposes of extrapolating our results or extending the system to other regions. Currently, the coverage of the system includes 214 laboratories in the PACA region, representing $81 \%$ of major urban areas of the PACA region (e.g., Marseille, Nice, and Toulon). Only 1 department of the region, the Hautes Alpes, is not properly covered.

The third strength is the diversity of bacterial species identified and transmitted by the network of laboratories in the PACA region. The number of samples tested and their diversity are greater in the PACASurvE than in other surveillance systems currently active in France (10). This difference could be explained by the higher number of participating laboratories, which increases continually. This high diversity underlines the relevance of a private-sector laboratory surveillance system operating in parallel with a hospital epidemiologic surveillance system. Currently, PACASurvE monitors only bacterial species, but it would be interesting to extend this surveillance to other subjects, such as antibiograms or viruses.

Our biosurveillance system has some limitations. The first relates to the statistical analyses used at the beginning for the detection of abnormal events. The use of a threshold of 2 SDs higher than the historical weekly mean is not necessarily appropriate, although it is easy to set up rapidly and enabled detection of an abnormal event. At the beginning of the surveillance system data collection, in the absence of strong historical data, this basic algorithm seemed to be the most appropriate and easy to use given the circumstances. After studying the methods used to address seasonal variations and sporadic emergence of rare bacterial species as described by Enki et al. (13) in 2013, Farrington et al. (28) in 1996, Buckeridge et al. (29) in 2004, and Frickers et al. (30) in 2008, we decided to implement another method and this introduced the $\mathrm{C} 1$-mild epidemics detection method (18) with R software into the surveillance package. This method is now used routinely in CESPA.

The second limitation concerns the laboratories' willingness to participate, which could lead to problems in reporting. For example, we have to encourage laboratories to report their data automatically by using the Internet platform.

After only 2 years in operation, the results achieved by our network are already promising. The economic cost of this system will be calculated, being a major criteria for the first planned evaluation of the system. In the future, we will improve the completeness of transmitted data and will try to extend our network to other regions in France. This type of regional biosurveillance network could be linked to data from existing networks implemented by the National Institute for Public Health Surveillance (Santé Publique France) to enable comprehensive surveillance of all French territory. An equivalent of the Epidemiologic Surveillance and Alert Based on Microbiological Data system has been set up in Senegal with the participation of several health centers. In conclusion, the recent development of a surveillance network based on data from microbiologic laboratories in the PACA region has demonstrated its value for early identification of regional epidemics.

\section{Acknowledgments}

We would like to thank Gaetan Texier for his help with the organization of his paper. We would also like to thank the additional members of PACASurvE who contributed data: Jean Paul Casalta, Pierre Yves Levy, Jean Pierre Arzouni, Philippe Halfon, Alexandre Toro, Patricia Roussellier, Raymond Ruimy, Philippe Seyral, Jean Dominique Poveda, Éric Garnotel, Patrick Brunet, Pierre Hance, Nathalie Brieu, Christophe Maruejouls, Olivier Ridoux, Evelyne Jean. 
This work was supported by the University Hospital Institute Méditerranée Infection and the Armed Forces Center for Epidemiology and Public Health.

Authors' contributions: M.H. wrote the manuscript. G.B. participated in the development of the technical analysis in $\mathrm{R}$ and corrected the manuscript. C.A., X.D., D.R. provided comments on the manuscript and final approval.

Dr. Huart was a PhD student at the Institut HospitaloUniversitaire Méditerranée Infection, Aix-Marseille Université, and at the Centre d'Epidémiologie et de Santé Publique des Armées. His research interest is the development of a system for epidemiologic surveillance of infectious diseases that uses data from microbiologic laboratories in the Provence-Alpes-Côte d'Azur region.

\section{References}

1. Raoult D. The causes underlying the emergence of disease agents [in French]. Annales des Mines-Responsabilité et Environnement. 2008;3:21-4. https://dx.doi.org/10.3917/re.051.0021

2. GBD 2013 Mortality and Causes of Death Collaborators. Global, regional, and national age-sex specific all-cause and cause-specific mortality for 240 causes of death, 1990-2013: a systematic analysis for the Global Burden of Disease Study 2013. Lancet. 2015;385:117-71. http://dx.doi.org/10.1016/ S0140-6736(14)61682-2

3. Declich S, Carter AO. Public health surveillance: historical origins, methods and evaluation. Bull World Health Organ. 1994;72:285-304.

4. M'ikanatha N, de Valk H, Lynfield R, Van Benden C. Infectious disease surveillance: a cornerstone for prevention and control. In: M'ikanatha N, de Valk H, Lynfield R, Van Benden C, editors. Introduction to infectious disease surveillance. 2nd ed. Oxford: John Wiley \& Sons; 2013. p. 1-20.

5. Morens DM, Folkers GK, Fauci AS. The challenge of emerging and re-emerging infectious diseases. Nature. 2004;430:242-9. http://dx.doi.org/10.1038/nature02759

6. De Cos H. Treatise on the airs, waters, and places. In: É Littré. Complete works of Hippocrates [in French]. Vol. 2. Paris: J.B. Baillière; 1840. p. 1-93.

7. Abat C, Chaudet H, Rolain J-M, Colson P, Raoult D. Traditional and syndromic surveillance of infectious diseases and pathogens. Int J Infect Dis. 2016;48:22-8. http://dx.doi.org/10.1016/j.ijid.2016.04.021

8. Buckeridge D. Detection of outbreaks using laboratory data: an epidemiological perspective [cited 2016 Dec 22]. https://www.inspq.qc.ca/sites/default/files/jasp/archives/2006/ jasp2006-laboratoire-lbuckeridge1.pdf

9. Colson P, Rolain J-M, Abat C, Charrel R, Fournier P-E, Raoult D. EPIMIC: a simple homemade computer program for real-time epidemiological surveillance and alert based on microbiological data. PLoS One. 2015;10:e0144178. http://dx.doi.org/10.1371/ journal.pone. 0144178

10. Abat C, Chaudet H, Colson P, Rolain J-M, Raoult D. Real-time microbiology laboratory surveillance system to detect abnormal events and emerging infections, Marseille, France. Emerg Infect Dis. 2015;21:1302-10.http://dx.doi.org/10.3201/ eid2108.141419

11. Seng P, Abat C, Rolain JM, Colson P, Lagier J-C, Gouriet F, et al. Identification of rare pathogenic bacteria in a clinical microbiology laboratory: impact of matrix-assisted laser desorption ionization-time of flight mass spectrometry. J Clin Microbiol. 2013;51:2182-94.http://dx.doi.org/10.1128/ JCM.00492-13

12. Plouin-Gaudon I, Vanhems P, Allard R, Sahajian F, Fabry J. Surveillance of laboratory based infections by biological and medical analyses: review of the literature [in French]. Sante Publique. 2000;12:149-59.

13. Enki DG, Noufaily A, Garthwaite PH, Andrews NJ, Charlett A, Lane C, et al. Automated biosurveillance data from England and Wales, 1991-2011. Emerg Infect Dis. 2013;19:35-42. http://dx.doi.org/10.3201/eid1901.120493

14. Walckiers D, Vandepitte J, Stroobant A, Cornelis R, Van Casteren VD, Walckiers JV. A new method of surveillance of infectious diseases in Belgium: the "Vigie" network of microbiology laboratories [in French]. Med Mal Infect. 1986; 16:147-50. http://dx.doi.org/10.1016/S0399-077X(86)80217-7

15. French National Institute of Statistics and Economic Studies. Legal population in force as of January 1, 2014: census of population [in French] [cited 2016 Apr 1]. https://www.insee.fr/fr/statistiques/ fichier/2119747/dep91.pdf

16. Hulth A, Andrews N, Ethelberg S, Dreesman J, Faensen D, van Pelt W, et al. Practical usage of computer-supported outbreak detection in five European countries. Euro Surveill. 2010;15:19658.

17. R Core Team. R: a language and environment for statistical computing [cited 2016 Dec 22]. http://www.R-project.org

18. Hutwagner L, Thompson W, Seeman GM, Treadwell T. The bioterrorism preparedness and response Early Aberration Reporting System (EARS). J Urban Health. 2003;80 (Suppl 1):i89-96.

19. Wang X, Zeng D, Seale H, Li S, Cheng H, Luan R, et al. Comparing early outbreak detection algorithms based on their optimized parameter values. J Biomed Inform. 2010;43:97-103. http://dx.doi.org/10.1016/j.jbi.2009.08.003

20. Abat C, Huart M, Garcia V, Dubourg G, Raoult D. Enterococcus faecalis urinary-tract infections: do they have a zoonotic origin? J Infect. 2016;2016:305-13. https://dx.doi.org/10.1016/ j.jinf.2016.07.012

21. Lagier J-C, Dubourg G, Cassir N, Fournier P-E, Colson P, Richet $\mathrm{H}$, et al. Clostridium difficile 027 emerging outbreak in Marseille, France. Infect Control Hosp Epidemiol. 2013;34:133941.http://dx.doi.org/10.1086/673995

22. Huart M, Abat C, Jimeno MT, Deparis X, Raoult D, Fournier P-E. Compared lethality rates of Clostridium difficile infections at the local, regional and national levels in France. New Microbes New Infect. 2016;14:6-7. http://dx.doi.org/ 10.1016/j.nmni.2016.07.006

23. Lagier J-C, Delord M, Million M, Parola P, Stein A, Brouqui P, et al. Dramatic reduction in Clostridium difficile ribotype 027associated mortality with early fecal transplantation by the nasogastric route: a preliminary report. Eur J Clin Microbiol Infect Dis. 2015;34:1597-601. https://dx.doi.org/10.1007/ s10096-015-2394-x

24. Walckiers D, Stroobant A, Yourassowsky E, Lion J, Cornelis R. A sentinel network of microbiological laboratories as a tool for surveillance of infectious diseases in Belgium. Epidemiol Infect. 1991;106:297-303.http://dx.doi.org/10.1017/ S0950268800048445

25. Kalish BT, Gaydos CA, Hsieh Y-H, Christensen BE, Carroll KC, Cannons A, et al. National survey of Laboratory Response Network sentinel laboratory preparedness. Disaster Med Public Health Prep. 2009;3(Suppl):S17-23. http://dx.doi.org/10.1097/DMP.0b013e31819e09b4

26. Walckiers D, Van Ros G, Stroobant A. Monitoring of malaria in Belgium through a network of microbiology laboratories [in French]. Ann Soc Belg Med Trop. 1986;66:15-21. 
27. Silva JC, Shah SC, Rumoro DP, Bayram JD, Hallock MM, Gibbs GS, et al. Comparing the accuracy of syndrome surveillance systems in detecting influenza-like illness: GUARDIAN vs. RODS vs. electronic medical record reports. Artif Intell Med. 2013;59:169-74.http://dx.doi.org/10.1016/j.artmed.2013.09.001

28. Farrington CP, Andrews NJ, Beale AD, Catchpole MA. A statistical algorithm for the early detection of outbreaks of infectious disease. J R Stat Soc Ser A Stat Soc. 1996;159:547-63. http://dx.doi.org/10.2307/2983331

29. Buckeridge DL, Burkom H, Campbell M, Hogan WR, Moore AW. Algorithms for rapid outbreak detection: a research synthesis.
J Biomed Inform. 2005;38:99-113. http://dx.doi.org/10.1016/j. jbi.2004.11.007

30. Fricker RD Jr, Hegler BL, Dunfee DA. Comparing syndromic surveillance detection methods: EARS' versus a CUSUM-based methodology. Stat Med. 2008;27:3407-29.

http://dx.doi.org/10.1002/sim.3197

Address for correspondence: Michael Huart, Centre d'Epidémiologie et de Santé Publique des Armées (CESPA), Camp de Sainte Marthe, 408 Rue Jean Queillau, 13014 Marseille, France; email: mikoli@hotmail.fr

\section{August 2015: Surveillance}

- Drivers of Emerging Infectious Disease Events as a Framework for Digital Detection

- Escherichia coli 0157 Outbreaks in the United States, 2003-2012

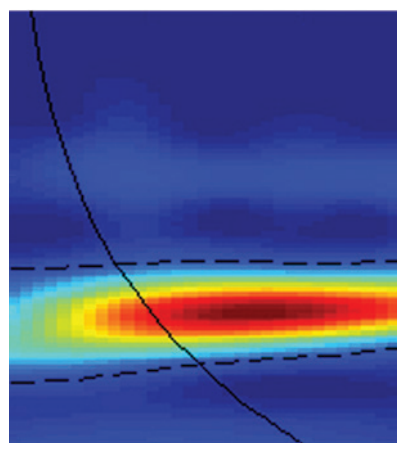

- Real-time Microbiology Laboratory Surveillance System to Detect Abnormal Events and Emerging Infections, Marseille, France

- Underrecognition of Dengue during 2013 Epidemic in Luanda, Angola

- Health Care-Associated Infection Outbreak Investigations in Outpatient Settings, Los Angeles County, California, USA, 2000-2012

- Response Strategies against Meningitis Epidemics after Elimination of Serogroup A Meningococci, Niger
- Phylogeography of Influenza A(H3N2) Virus in Peru, 2010-2012

- Influenza A Viruses of Human Origin in Swine, Brazil

- Differentiation of Acute Q Fever from Other Infections in Patients Presenting to Hospitals, the Netherlands

- Susceptibility of Carrion Crows to Experimental Infection with Lineage 1 and 2 West Nile Viruses

- Hospital Resource Utilization and Patient Outcomes Associated with Respiratory Viral Testing in Hospitalized Patients

- Development of Framework for Assessing Influenza Virus Pandemic Risk

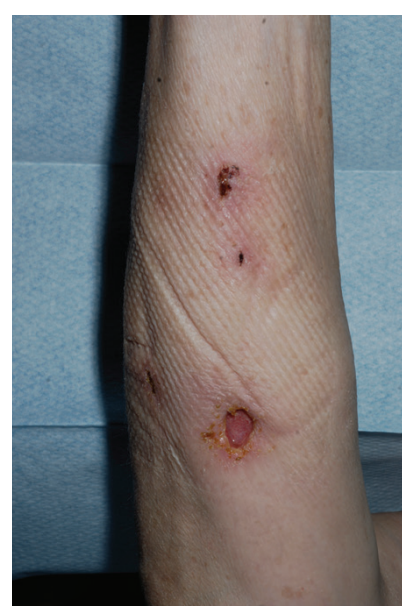

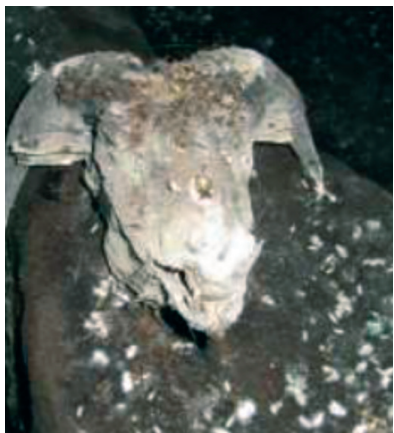

- Community-Based Outbreak of Neisseria meningitidis Serogroup C Infection in Men who Have Sex with Men, New York City, New York, USA, 2010-2013

- Risk for Mycobacterial Disease among Patients with Rheumatoid Arthritis, Taiwan, 2001-2011

- Prevalence of Hepatitis E Virus Infection in Pigs at the Time of Slaughter, United Kingdom, 2013

- Estimates of Outbreak Risk from New Introductions of Ebola with Immediate and Delayed Transmission Control

- Genomic Assays for Identification of Chikungunya Virus in Blood Donors, Puerto Rico, 2014

- Seasonal Patterns of Buruli Ulcer Incidence, Central Africa, 2002-2012
- Human-Bat Interactions in Rural West Africa

- Occupational Exposure to Dromedaries and Risk for MERS-CoV Infection, Qatar, 2013-2014

- Cutaneous Legionella longbeachae Infection in Immunosuppressed Woman, United Kingdom

- Bartonella spp. and Coxiella burnetii Associated with Community-Acquired, Culture-Negative Endocarditis, Brazil

- Detection and Full-Length Genome Characterization of Novel Canine Vesiviruses

- Smallpox Vaccination of Laboratory Workers at US Variola Testing Sites

- Enterovirus A71 Meningoencephalitis Outbreak, Rostov-on-Don, Russia, 2013

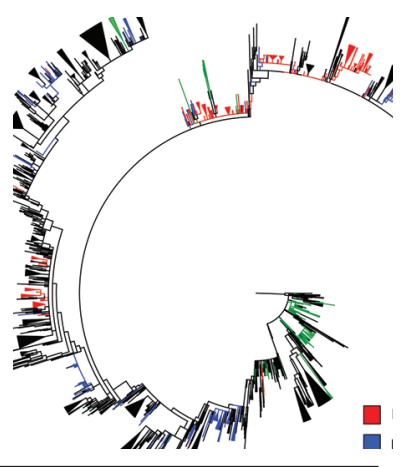

EMERGING INFECTIOUS DISEASES http://wwwnc.colc.gov/eid/articles/ issue $/ 21 / 8 /$ table-of-contents 